\title{
SOPHIE CHÉRON
}

\author{
Université Adam Mickiewicz, Poznań
}

\section{LE TRIO HERGÉEN : UN TOURNANT DANS LES AVENTURES DE TINTIN}

\begin{abstract}
Chéron Sophie, Le trio hergéen : un tournant dans "Les aventures de Tintin » [The Hergéan trio: A turning point in The Adventures of Tintin],Studia Romanica Posnaniensia, Adam Mickiewicz University Press, Poznań, vol. XXXIX/1: 2012, pp. 89-98, ISBN 978-83-232-2410-5, ISSN 0137-2475, eISSN 2084-4158.

Before Captain Haddocks' and Professor Calculus' appearance in The Crab with the Golden Claws (eighth album, 1941) and Red Rackham's Treasure (eleventh album, 1944) Tintin doesn't have strong links with the other characters. His youth, dynamism and resourcefulness are his only characteristics - he has no past, no name, no family, no occupational ties. The two new friends Hergé gives him provide the last ten albums of The Adventures more consistency, humor, and a proper space which were missing until then.
\end{abstract}

Keywords. comics, Hergé, Tintin, identity, narrative function

Tintin est une variante de ce que Marc Quaghebeur appelle « la figure mythique du petit belge héroïque ${ }^{1} \gg$. Le jeune reporter est un personnage d'action solitaire qui se distingue d'une part par ses actes et son dynamisme et d'autre part, au niveau diégétique, par sa capacité à faire avancer l'intrigue, à amorcer les aventures tout en restant modeste par rapport aux actes de bravoure accomplis. Dans la première partie des albums (de Tintin au Congo au Crabe aux Pinces d'Or) l'univers hergéen est encore flou et la complexité psychologique de Tintin est trop faible pour supporter un dense ensemble d'intrigues. Hergé donne un souffle nouveau à ses aventures en $\mathrm{y}$ introduisant deux personnages plus étoffés psychologiquement et plus solidement inscrits dans le réseau des personnages secondaires, qui gravite désormais autour d'eux : il s'agit du capitaine Haddock et du professeur Tournesol. Leur apparition dans les aventures donne à la deuxième partie des albums (du Crabe aux Pinces d'Or

${ }^{1}$ Cette figure, « foncièrement jeune, dont les comportements sont plein de noblesse, d'impertinence et de débrouillardise » est apparue dans le mythe du XVI ème siècle exploité dans la littérature belge francophone dès le XIX ${ }^{\text {ème }}$ siècle. Les Pays-Bas espagnols du XVI ${ }^{\text {ème }}$ siècle étaient soumis à la tyrannie d'une puissance dévastatrice pour les dix-sept provinces. Marc Quaghebeur a par ailleurs montré que cette mythification historique se développe avant l'indépendance politique de la Belgique de 1830 (avec Le Gueux de mer de Henri Moke en 1827). La Légende de Thyl Ulenspiegel de Charles de Coster donna au mythe puissance et pérennité dans la littérature belge de langue française. 
à Tintin et les Picaros) une vitalité nouvelle et une cohérence plus profonde dans la progression des intrigues. Dans cet article, il s'agit d'abord de montrer que la complexité psychologique de Tintin, son ancrage dans le réseau de personnages secondaires et dans l'espace géographique sont insuffisants pour soutenir un renouvellement cohérent des aventures. Je montre ensuite que l'apparition de Haddock et Tournesol (dans les $9^{\text {ème }}$ et $11^{\text {ème }}$ albums) est un moment charnière qui restructure l'univers hergéen et lui donne une dimension historique et humoristique.

\section{TINTIN, UN MOTEUR D'ACTION}

Tintin est d'abord un personnage d'action. Sur le plan graphique, le style de la ligne claire accentue cette caractéristique qui est déjà très présente au niveau de la composition psychologique du personnage. Les aventures de Tintin sont aujourd'hui l'emblème du style de la ligne claire, dont Hergé est la figure principale depuis les années 50. L'école, qui domine jusque dans les années 60 , se caractérise par une clarté graphique et narrative : dessins sans détail superflu ni hachure et lisibilité de l'intrigue y sont d'usage. Les comics et le cinéma américains servirent de modèles pour ce nouveau style narratif. Hergé précise en effet qu'

une des qualités essentielles de la bande dessinée américaine, comme d'ailleurs du cinéma américain, [lui] parait être sa grande clarté. En général, les américains savent raconter une histoire, même si cette histoire est une cornichonnerie. C'est là, [croit-il], la grande leçon [qu'il a] tirée, à la fois de la bande dessinée et du cinéma américain ${ }^{2}$.

Deux autres caractéristiques de ce style, propres aussi au cinéma américain, sont la vitesse et le mouvement. La bande dessinée autorise facilement l'ellipse tout en laissant visuellement en présence l'avant et l'après, ce qui accentue l'effet de mobilité. Par ailleurs il n'est pas rare de voir Tintin passer de son lit à la cage d'escalier, enfilant son manteau à la va-vite, ou du restaurant d'un hôtel à un aéroport étranger, entraînant avec lui ses amis à travers le monde.

Tintin lui-même hérite de ces caractéristiques sur le plan narratif car c'est lui qui fait avancer l'action. Un exemple issu du Secret de la Licorne permet de le mettre en lumière : le capitaine vient de découvrir dans une malle de son grenier les mémoires de son ancêtre le Chevalier de Hadoque, commandant du vaisseau La Licorne sous le roi Louis XIV. Lorsque Tintin lui rend visite le lendemain, il lui rapporte l'abordage du navire par le pirate Rackham le Rouge en ponctuant son récit de plusieurs verres de whisky. Agacé par l'abus d'alcool de son camarade, Tintin l'empêche de boire et lui

${ }^{2}$ Cité dans J.-L. Tilleuil, « La bande dessinée, une histoire belge ? », Ch. Berg, P. Halen, Littératures belges de langue française. Histoire et perspectives (1830-2000), Le Cri, coll. « Histoire », Bruxelles 2000, p. 567-594. 
demande plusieurs fois de poursuivre son récit d'aventure qu'il écoute attentivement $\left(22 \mathrm{C}\right.$ et $\left.\mathrm{D}^{3}\right)$.

[Haddock] - La nuit venue, les pirates ayant découvert les tonneaux de rhum dans les cales de la Licorne, les mirent en perce et n'enivrèrent abominablement... [...] Abominablement... Oui, abominablement, c'est le mot. (il prend un verre de whisky, que Tintin lui enlève des mains) Eh bien quoi ?... Je voulais simplement vous montrer...

[Tintin, déposant le verre sur le sol] - Inutile capitaine, j'ai très bien compris.

[Haddock] - comme vous voudrez, Tintin... Où en étais-je ?

[Tintin] - Au moment où les pirates s'enivraient abominablement...

Sur le plan psychologique, l'action est également un facteur déterminant le comportement du héros. Tintin se définit exclusivement par sa jeunesse, sa noblesse de cœur et sa débrouillardise. Il est toujours en quête d'aventures, de chasses au trésor, d'énigmes à résoudre et de mystères à dévoiler". Il n'abandonne jamais ses amis, qu'il n'hésite pas à arracher des griffes du yéti tibétain ${ }^{5}$, qu'il sauve de l'alcoolisme ${ }^{6}$, qu'il accompagne sur les traces de leur histoire ${ }^{7}$. Il trouve toujours un moyen, parfois modérément crédible ${ }^{8}, d^{\prime}$ 'échapper aux multiples pièges et embuches qui encombrent son chemin. Tintin n'existe que par ses actes. S'il est journaliste, on ne le voit presque jamais écrire - par contre il est capable de piloter un avion, d'envoyer des messages en morse, de diriger une fusée ou un bateau.

Ses aventures sont toujours amorcées par une anecdote : un bateau miniature découvert sur un marché aux puces, une lettre envoyée par un ami. Comme le remarque Benoit Peeters, « Hergé entre toujours dans le récit par la petite porte (une serviette oubliée, une boîte de crabe dans une poubelle) au plus près du quotidien. Il conclut

${ }^{3}$ Pour des raisons d'impression, il n'a pas été possible d'ajouter d'images à cet article. Les références aux albums suivent le modèle suivant : le titre de l'album, un numéro en chiffre romain pour la planche, une lettre en majuscule pour la bande et un second numéro en chiffre romain pour la case. Les abréviations Licorne et Trésor correspondent respectivement aux albums Le Secret de la Licorne (1943) et Le Trésor de Rackham le Rouge (1944).

${ }^{4}$ Dans Le secret de la Licorne et Le trésor de Rackham le Rouge, il vole au secours de ses amis les Dupondt incapables de découvrir l'identité du pickpocket qui sévit dangereusement; il construit un bélier avec de simples draps de lit pour s'évader de la crypte où il est retenu prisonnier par deux malfrats ; il assemble les trois indices essentiels à la découverte du trésor d'un pirate ; il conduit un sous-marin ; et il résout l'énigme mystérieuse du Chevalier, s'appropriant ainsi un coffret de bijoux vieux de plusieurs siècles.

${ }^{5}$ Hergé, Tintin au Tibet, Casterman, Bruxelles 1960.

${ }^{6}$ Hergé, Tintin et le Crabe aux Pinces d'Or, Casterman, Bruxelles 1943 (première édition en 1941).

${ }^{7}$ Hergé, Le trésor de Rackham le Rouge, Casterman, Bruxelles 1944.

${ }^{8}$ Pour s'échapper d'une crypte dans laquelle il est retenu prisonnier, Tintin construit par exemple un bélier en fixant une poutre à des draps de lit noués bout à bout. Il utilise également un caillou et un bout de ficelle pour soulever la poutre et la pendre au plafond, en passant la corde de draps à travers un anneau fixé au plafond. Tintin découvre tout ce matériel sur place et parvient à mettre son plan à exécution en moins de deux heures (Le trésor de Rackham le Rouge, 36 à 39). 
par un gag ou une pirouette, jamais par une brabançonne $»^{9}$. Tintin ne se pose pas en défenseur de justice ou de paix, comme les super-héros américains. Il n'est en quête ni de gloire ni de reconnaissance. Lorsqu'il est face à un conflit, il prend toujours le rôle du modérateur pacifique ferme mais conciliant sans recourt à la violence. Jamais dans les albums Hergé ne met ses lecteurs face à une scène d'agressivité. Les coups sont la plupart du temps elliptiques : on y voit l'agresseur prêt à frapper directement suivi d'une victime assommée et d'aimables petites étoiles multicolores.

Tintin est pourtant un personnage inconsistant : il n'a pas de nom, pas de passé, pas d'attaches familiales ou professionnelles et mis à part son grand cœur, sa jeunesse et son sens de l'aventure aucun trait distinctif ne le caractérise. Il s'exprime dans un français neutre et ampoulé qui ne colle pas avec le spontané des situations dans lesquelles il se trouve. Il n'est pas non plus défini par une appartenance sexuelle : ses traits ne sont pas spécialement masculins et il n'est jamais en contact avec les femmes. Lorsqu'elles sont représentées, elles sont toujours un prétexte de caricature. Il en va ainsi de la Castafiore, chanteuse manquée, grosse, laide et trop sûre de son succès, mais également d'autres personnages secondaires. Dans Le Secret de la Licorne, les trois femmes qui sont représentées sont une bourgeoise mal élevée qui occupe la cabine téléphonique publique pour se protéger de la pluie, alors que Tintin attend à l'extérieur pour passer un coup de téléphone urgent ( 8 et 9); une passante curieuse et peureuse ${ }^{10}$ informant Tintin et Haddock de la mort de M. Sakharine, alors que ce dernier n'est qu'évanoui (28 et 29) ; et la concierge de Tintin trop stupide pour réaliser que celui-ci vient d'être kidnappé sous son nez, alors qu'elle a tous les indices en main pour le deviner (35 et 36 ).

Tintin n'a pas non plus de lieu propre. Il est de Bruxelles mais on l'y voit très peu, et il a sillonné plusieurs continents sans que l'on sache d'où lui viennent le temps et l'argent qu'il consacre à tous ses voyages. Daniel Justens et Alain Préaux ${ }^{11}$ ont montré l'importance de l'ancrage bruxellois des Aventures par l'étude des détails picturaux et du dialecte bruxellois, le marollien, dans les albums. On constate avec eux que l'utilisation du dialecte bruxellois s'intensifie au fil de la série. Ces régionalismes sont utilisés par d'autres personnages pour créer une langue exotique (comme c'est le cas du syldave dans Tintin et le sceptre d'Ottokar ${ }^{12}$ ). Par ailleurs, certaines références spatiales restent présentes de manière anecdotique : un ancien timbre d'un franc belge sur une lettre adressée aux frères Loiseau (Le Secret de la Licorne, 45A2), une allusion à un village du Brabant wallon (même planche, 45D1) ou au Théâtre

${ }^{9}$ B. Peeters, « 1929 - Naissance de Tintin » in J.-P. Bertrand, R. Grutman, B. Denis et M. Biron (dir.), Histoire de la littérature belge francophone (1830-2000), Fayard, Paris 2003.

${ }^{10}$ Elle écoute aux portes et s'enfuit, effrayée par les insultes du capitaine Haddock.

${ }^{11}$ D. Justens, A. Préaux, Tintin, ketje (gamin) de Bruxelles, Casterman, Bruxelles 2004.

${ }^{12}$ Dans une interview avec Benoit Peeters, Hergé confirme s'être « amusé » à se servir du marrollien pour créer le syldave. «C'était un petit clin d'œil que j'adressais au Bruxellois. Et de toute façon, ça ne pouvait rien enlever aux autres lecteurs, au contraire » précise-t-il. (Le monde d'Hergé, Bibliothèque de Moulinsart, Casterman, Bruxelles 1990). 
des Galeries bruxellois (Le Trésor de Rackham le Rouge, 2C1). Ces références sont toujours subtiles, tandis que les références explicites à la Belgique très présentes dans les premiers albums s'effacent progressivement. Ainsi, la leçon de géographie belge de la première édition de Tintin au Congo (1931) disparaît lors de la parution de l'album en couleur en 1946 pour donner lieu à une leçon d'arithmétique ${ }^{13}$. De même, il n'est plus jamais explicitement question de reportage pour le Petit Vingtième bruxellois après le premier album (Tintin au pays des Soviets), même si les premières versions des planches continuent à être éditées dans le magazine jusqu'à la fin de sa diffusion en mai 1940.

En conclusion, Tintin apparaît sur les plans de l'intrigue et du dessin comme un véhicule narratif ${ }^{14}$ sur lequel reposent le mouvement, la vitesse, l'énergie des aventures. Sur le plan psychologique, il se présente comme un personnage d'action sans véritable profondeur : il est seul, sans attache, ses liens avec les autres personnages secondaires sont ténus.

\section{LE TRIO HERGÉEN}

Cette solitude, ce détachement par rapport à un lieu propre et au réseau des personnages secondaires affaiblissent la cohérence des albums et auraient pu épuiser progressivement les aventures ou les rendre ennuyeuses et répétitives. La création d'un trio de personnages soudés les uns aux autres permet à Hergé de restructurer l'univers des albums sans tomber dans cette impasse. L'apparition du capitaine dans le huitième volume (Le Crabe aux Pinces d'Or) et de Tournesol dans le onzième (Le trésor de Rackham le Rouge), leurs liens avec le réseau des personnages secondaires et leur ancrage dans le château de Moulinsart est un véritable tour de force de la part d'Hergé, qui a désormais doté sa série d'un nouveau potentiel narratif.

Dans Le Secret de la Licorne, au cœur de cette transition, Tintin est mis en retrait pour la première fois du point de vue de l'action ${ }^{15}$. L'attention se focalise sur le Chevalier de Hadoque qui a caché les bijoux espagnols et sur les deux nouveaux amis du héros, qui ont en main les clés pour découvrir le coffret de diamants (Licorne, 21D3). C'est en effet l'aptitude du capitaine à manœuvrer le Sirius et à s'orienter sur les eaux ainsi

${ }^{13}$ Dans la première édition de Tintin au Congo parue dans « Le Petit Vingtième » en 1930-1931, Tintin donne une leçon de géographie belge à une classe de jeunes congolais en leur parlant de « leur patrie».

14 Pour reprendre l'expression de Benoît Peeters dans « 1929 - Naissance de Tintin », op. cit.

15 «La première place dans les aventures n' [...] appartient plus [à Tintin]. Elle revient au chevalier François de Hadoque, véritable clé de voute de l'univers d'Hergé, dont le fantôme ne cessera de hanter l'univers de Moulinsart. Cette aventure permet enfin à Tintin d'échapper à la gémellité, qui était le principal danger rencontré dans la phase épique de ses aventures, c'est-à-dire jusqu'au Sceptre d'Ottokar » (Apos tolidès, « dans le ventre de la licorne », A. Algoud, J.-M. Apostolidès, D. Cerbelaud, B. Peeters, P. Sterckx, L'Archipel Tintin, Essai / coll. « Réflexions faites », 2004, p. 84). 
que l'invention du sous-marin par le professeur qui permettent de découvrir l'épave de la Licorne. Qui plus est, l'argent que Tournesol reçoit du gouvernement pour le brevet de ce même submersible sera utilisé par les trois compagnons pour racheter le château de Moulinsart et faire entrer par la même occasion le majordome Nestor, dévoué à ses nouveaux maîtres, dans le réseau des personnages secondaires.

Le Crabe aux Pinces d'Or et Le Secret de la Licorne présentent le capitaine Haddock comme un ancien alcoolique qui a par le passé été en contact avec d'autres personnages (le lieutenant Thompson Allan par exemple, déjà présent dans le troisième album Les Cigares du Pharaon), mais surtout comme l'ancêtre d'un illustre capitaine de la marine française dont l'image sera sans cesse présente au château de Moulinsart. Il s'agit d'un Chevalier français du XVII ${ }^{\mathrm{ème}}$ siècle digne, rusé, adroit, courageux et parfaitement apte à garder son sang-froid. Les ressemblances et les divergences de Haddock avec son ancêtre lui donnent une profondeur psychologique qui détermine ses réactions dans les Aventures.

Dans l'histoire de ses aventures racontées à Tintin (14 à 26), l'alternance entre le discours du capitaine Haddock et l'aventure de François de Hadoque lui-même met l'accent sur les correspondances entre les deux personnages. Les récits de l'un et de l'autre s'enchaînent parfaitement (Licorne, $16 \mathrm{C} 1$ et 2) et la proximité des deux scènes présente et passée facilite l'identification de Haddock avec son ancêtre, qui finissent tous deux par ne faire qu'un lorsqu'Haddock se retrouve la tête enfoncée au travers du portrait de son aïeul (Licorne, 25B et C).

Leurs apparences physiques sont quasi identiques. Seuls leur coiffure et leurs vêtements, dont les couleurs restent proches (bleu et mauve), changent. Qui plus est, le capitaine s'approprie consciemment les attributs de son ancêtre : son chapeau à plume, ses faits et gestes, ses qualités. Il veut donner l'image d'un homme courageux et intrépide alors qu'il est en réalité plutôt casanier et attaché à son confort. Les accusations injustes et les soupçons de couardise ou de poltronnerie à son égard sont avec l'alcool le moyen le plus efficace de l'entraîner sur les routes du mystère et du danger. Dans Le Trésor, il s'apprête à annuler son départ à la recherche de la Licorne prétextant diverses excuses (10 à 12):

[Dupond] - Est-ce vrai, capitaine ? Tintin vient de nous dire que vous aviez décidé de ne pas partir. Il paraît que vous avez brisé un miroir et que vous avez peur de...

[Haddock] - Peur ?... Moi peur ?... Peur de quoi ?... Peur de qui ?... Peur de vous peut-être, hein ?... Apprenez que le capitaine Haddock n'a peur de rien ! Compris ?... Demain, à l'aube, nous levons l'ancre !... Qu'on se le dise !

Pourtant, l'identification totale n'est qu'apparente. De manière générale, Haddock se présente comme une version démythifiée de son ancêtre ; son univers est basé avant tout sur quelques plaisirs et de simples valeurs comme l'amitié plutôt que sur un illustre nom à défendre et sur un ennemi juré à combattre. Il se distancie aussi de son aïeul par des traits de caractère qui lui sont propres : son penchant pour l'alcool, 
sa maladresse, sa malchance, ses emportements coléreux. La double scène du combat final contre Rackham le Rouge (Licorne, 25 et 26) rend patente la divergence entre les deux parents. Le capitaine Haddock, rougi par l'alcool, se bat maladroitement contre un ennemi imaginaire de manière visiblement inefficace (Licorne, 25B2) ; il détruit tout sur son passage et effraye même le chien Milou. Le Chevalier, sobre au contraire, maîtrise l'art de l'escrime contre un véritable ennemi qu'il finit par achever dignement. La scène de violence finale est seulement sous-entendue verbalement : « et voilà !... Dieu lui pardonne tous ces crimes ».

La différence entre les deux capitaines est également visible lorsque l'on compare leur manière d'insulter leurs opposants. D'un côté le Chevalier de Haddoque vainc physiquement et verbalement son ennemi ; de l'autre, le capitaine n'effraye personne par ses insultes et il a tendance à fuir les dangers.

François de Hadoque se présente en effet comme l'antagoniste absolu de son ennemi Rackham le Rouge. L'un tente de sauver puis de venger ses hommes ; 1'autre est un pillard diabolique invoquant Lucifer (Licorne, 24C1), associé au rouge (par son nom et la couleur de ses vêtements) et portant une barbiche noire. Le duel entre les deux ennemis (Licorne, 24) se double d'un combat verbal lors duquel Hadoque réplique aux attaques verbales de son ennemi de manière cohérente : il le traite de « perroquet bavard », de « flibustier de carnaval », de « pirate d'eau douce » et d' " anthropopithèque », allusions à ses paroles inutiles, son costume d'apparat, son incompétence, sa barbarie. Ces insultes réduisent l'ennemi au silence. Finalement, Hadoque gagne physiquement et verbalement le combat : Rackham est laissé pour compte suite à l'insulte finale « vieux cachalot», qui reste en lien avec le lexique marin.

Le capitaine Haddock hérite apparemment des insultes de son ancêtre, qui l'ont par ailleurs rendu célèbre. L'usage qu'il en fait ne fonctionne pourtant pas de la même manière qu'avec le Chevalier. D'abord, les ennemis de Haddock sont généralement des opposants hors de portée (trop éloignés de lui ou dans l'impossibilité de répondre) alors que celui de son aïeul était proche et dangereux. Ensuite, les insultes du capitaine sont toujours inefficaces : si elles effrayent, c'est plus par leur expressivité que par leur signification. Elles sont sélectionnées en vrac selon leur aspect sonore, leur décalage contextuel et leur vide sémantique - toute réplique cohérente est donc rendue impossible. Haddock détourne donc les mots de leur sens et les dote d'un ton particulier : l'emportement, la colère, l'excès qui la caractérisent. Il copie la forme apparente des insultes de son ancêtre sans en maîtriser la logique ad hoc.

Le professeur Tournesol complète le trio à partir du onzième album, Le Trésor de Rackham le Rouge. Dès son entrée en jeu, il se présente comme celui qui a déjà inventé une série de machines (un nouveau modèle de gazogène, un appareil à brosser les vêtements, un lit placard) auxquelles il se plaît à faire allusion. Ayant eu vent de l'expédition de Haddock et Tintin pour découvrir le trésor dans les fonds marins, il se présente chez le jeune reporter pour lui proposer ses services de scientifique. 
Il vient d'inventer un submersible que les deux compagnons pourraient utiliser pour s'aventurer sous l'eau sans craindre les requins. Tryphon est sourd, myope et distrait. Il répond sans cesse à côté des questions soit qu'il ne les comprenne pas correctement, soit qu'il préfère les ignorer. Il utilise en effet cette stratégie pour s'imposer ou au contraire pour se dégager des situations qui l'importunent. Il réussit d'abord à entrer dans l'appartement de Tintin alors que Haddock vient d'éloigner une foule d'indésirables prétendants à leur part du trésor (Trésor, 5). Ensuite, il parvient à emmener Tintin, Haddock et les Dupondt dans son laboratoire pour leur montrer sa nouvelle invention malgré plusieurs refus explicites (Trésor, 6). Finalement, bien que Haddock ait écrit « votre appareil ne nous intéresse pas » sur un mur et sous ses yeux, le professeur prend l'initiative de l'embarquer à bord du navire, dans des caisses dont il a liquidé le contenu sans ménage (Trésor, 20). Ses idées paraissent parfois saugrenues mais se révèlent souvent brillantes et essentielles pour l'avancement de l'intrigue : c'est lui par exemple qui trouve le moyen de sauver Tintin du sous-marin coincé sous l'eau (Trésor, 26) ou qui déchiffre le manuscrit révélant que le château de Moulinsart appartient à la famille de Haddock (Trésor, 57C4). Son ingéniosité est par ailleurs la seule chose qu'il ne puisse entendre critiquer. Dans Objectif lune, il entre dans une colère mémorable parce que Haddock l'a traité de « zouave».

La surdité de Tryphon est ambiguë : il dispose d'un appareil acoustique dans Objectif lune et On a marché sur la lune, mais pas dans les autres albums. La surdité est utilisée par Hergé comme moteur d'action (notamment dans L'affaire Tournesol, où le professeur n'entend pas le coup de téléphone de Tintin qui tente de l'avertir d'une menace d'enlèvement) et comme moteur comique. Le désarroi dans lequel il plonge ses interlocuteurs en est un élément important. Dans Le Trésor, Haddock utilise cette situation pour se débarrasser d'un journaliste trop curieux, qu'il envoie auprès de Tournesol afin que celui-ci réponde à ses questions (Trésor, 56 et 57). Le journaliste est laissé pour compte après cinq répliques, au plus grand amusement du capitaine qui observe discrètement la scène (Trésor, 57A2). Tryphon s'en va alors en toute simplicité, en ayant écourté au maximum l'entretien déplaisant.

La compréhension décalée du professeur, anticipée ou à retardement, donne aussi une dimension humoristique aux Aventures, spécialement lorsqu'elle est confrontée aux réactions mesquines du capitaine Haddock - qui choisit souvent de se moquer de son ami plutôt que de se laisser déstabiliser et de se perdre en répétitions inutiles. Dans Le Trésor, le professeur découvre grâce à son pendule que les bijoux du Chevalier français ne se trouvent pas à l'endroit où Tintin et ses amis les cherchent. Il en avertit immédiatement le capitaine, qui ne le prend pas au sérieux. Celui-ci utilise le motif de l'arroseur arrosé, qui ne peut manquer de faire sourire le lecteur, contre Tournesol en répondant par la suite à côté des questions qu'il pose (Trésor, 41B, C et D) :

[Tournesol] - Dites-moi capitaine, je ne comprends pas... Puisque la Licorne ne se trouve pas ici, pourquoi Tintin est-t-il descendu ? 
[Haddock] - Il est allé cueillir des pâquerettes.

[Tournesol] - Une barquette ?... Où est-elle cette barquette ?...

(Tintin remonte à la surface en scaphandrier après avoir visité l'épave du bateau, découvert le jour même)

[Tournesol vexé et perplexe] - Pourquoi, mais pourquoi m'a-t-il dit que Tintin était parti en barquette?

Finalement, Tryphon découvrira le trésor grâce à son pendule dans la crypte de Moulinsart sous les yeux ébahis de Tintin et Haddock qui viennent eux-mêmes en prendre possession.

Tournesol et Haddock fonctionnent donc en miroir : les défauts de l'un sont moqués ou désamorcés par l'autre. En répondant à côté des questions, le professeur se pose en interlocuteur sur lequel les réponses sensées n'ont pas prise, de la même manière que les insultes du capitaine sont dénuées de signification et empêchent la réplique. Par ailleurs, le capitaine est le seul à moquer ouvertement le professeur et à utiliser sa surdité comme ressort de l'intrigue (dans le cas du journaliste, par exemple - Trésor, 56 et 57). Une autre fonction essentielle de Haddock est celle de garde-fou : il prévient Tintin de la folie de ses expéditions, souvent décidées sur un coup de tête. Par là, il pose un regard critique sur l'enthousiasme et la détermination absolue de son ami et il met à distance la perfection qui le rend sans doute invraisemblable.

\section{CONCLUSION}

Tintin est le moteur principal de l'action dans les premiers albums. Sa jeunesse, son dynamisme, sa noblesse de cœur, sa débrouillardise en font une autre figure du petit belge héroïque - figure déjà présente dans la littérature francophone de Belgique depuis Charles de Coster, qui éleva Thyl Ulenspiegel au rang de mythe littéraire. Néanmoins, la complexité psychologique de Tintin est assez faible et il est peu ancré dans le réseau des personnages. Ses deux nouveaux amis au contraire sont nettement plus complexes sur le plan psychologique. Ils ont un passé, un nom, des défauts (l'emportement et l'étourderie), une fonction sociale (un marin et un inventeur) et leurs réactions sont déterminées par leur identité. Haddock ne supporte pas de voir souillé l'honneur de son intrépide et valeureux ancêtre ; l'ingéniosité de Tournesol est régulièrement mise au service de l'avancement de l'intrigue. Par ailleurs, les deux nouveaux personnages dotent les aventures d'une dimension humoristique et d'un espace propre : le château de Moulinsart, héritage légitime du capitaine est acquis financièrement par le professeur. Dorénavant, c'est-à-dire à partir du diptyque Le Secret de la Licorne et Le Trésor de Rackham le Rouge et pour les dix albums suivants, les nouvelles aventures prennent en consistance car elles reposent sur Haddock et Tournesol. 


\section{BIBLIOGRAPHIE}

Apostolidès, J.-M. (2004). « Dans le ventre de la licorne » in A. Algoud, J.-M. Apostolidès, D. Cerbelaud, B. Peeters et P. Sterckx, L'Archipel Tintin. Essai, coll. « Réflexions faites ».

Justens, D. et Préaux, A. (2004). Tintin, ketje (gamin) de Bruxelles, Bruxelles : Casterman.

Peeters, B. (1990), Le monde d'Hergé, Bruxelles : Casterman, « Bibliothèque de Moulinsart».

Peeters, B. (2003). « 1929 - Naissance de Tintin » in J.-P. Bertrand, R. Grutman, B. Denis, M. Biron (dir.), Histoire de la littérature belge francophone (1830-2000), Paris : Fayard.

Quaghebeur, M. (2012). Permanence et Avatars du mythe du XVI siècle (à paraître) : Confluencias.

Tilleuil, J.-L. (2000). « La bande dessinée, une histoire belge ? » in Ch. Berg, P. Halen (dir.), Littératures belges de langue française. Histoire et perspectives (1830-2000), Bruxelles : Le Cri, coll. « Histoire ». 